\title{
Bioactive Polysaccharides of American Ginseng Panax quinquefolius L. in Modulation of Immune Function: Phytochemical and Pharmacological Characterization
}

Edmund M. K. Lui, Chike G. Azike, José A. Guerrero-Analco, Ahmad A. Romeh, Hua Pei, Sherif J. Kaldas, John T. Arnason and Paul A. Charpentier

Additional information is available at the end of the chapter

http://dx.doi.org/10.5772/50741

\section{Introduction}

Ginseng has a long history of use as a traditional medicine; and it is one of the top selling medicinal herbs in the world. It is a multi-action herb with a wide range of pharmacological effects on the central nervous system, cardiovascular system and endocrine secretion, and the reproductive and immune systems [1]. Ginseng is a deciduous, perennial plant of the Araliaceae family. There are two major species of ginseng: Panax ginseng and Panax quinquefolius; and the roots are primarily used for medicinal benefits. Ginseng's wide range of pharmacological activities is believed to be due to the presence of a host of bioactive compounds. The primary ones are the ginsenosides, which are steroidal saponins conjugated to different sugar moieties and polysaccharides (PS) which account for $10-20 \%$ by weight of ginseng.

Polysaccharide components of ginseng have received much attention recently because of the emergence of different biological activities, such as immunomodulatory, antibacterial, antimutagenic, radioprotective, anti-oxidative, anti-ulcer, antidepressant, anti-septicaemic and anti-inflammatory activities [2]. Specifically, the polysaccharide fraction of ginseng has been shown to have immunomodulatory effects in both preclinical and clinical studies [3-6], although they are poorly characterized.

Several polysaccharides have been identified in P. ginseng and P. notoginseng but these compounds, including arabinogalactan, pectins, and acidic polysaccharides, have been 
rarely studied in the North American species. They are made up of a complex chain of monosaccharides rich in L-arabinose, D-galactose, L-rhamnose, D-galacturonic acid, Dglucuronic acid and D-galactosyl residues [7]. The actual structural characteristics and the heterogeneity of PS components are poorly understood due to a lack of methodologies for separation as well as quantitative and qualitative analysis.

Most studies have focused on Asian ginseng PS and mostly in vitro experimental models. In this chapter, we will focus on Panax quinquefolius (American ginseng). The use of gel permeation chromatography (GPC) with multiple detectors has provided enhanced resolution for phytochemical analysis. And we have used both in vivo and in vitro models to evaluate its immunomodulatory activity.

\section{Materials}

Ginseng. Four-year-old American ginseng roots collected in 2007 from five different farms in Ontario, Canada were provided by the Ontario Ginseng Growers Association. Ginseng extracts from each farm were prepared individually and combined to produce composite extracts which were used for phytochemical and pharmacological studies [6]. The AQ extract has no detectable endotoxin contamination as determined by Limulus test.

\subsection{Chemicals and biologicals}

Sephadex G75 was purchased from GE Healthcare Bio-Sciences AB (Sweden). The Diethylaminoethyl (DEAE)-Cellulose and monosaccharide standards were purchased from Sigma (Oakville, Ontario). All other chemicals were of analytical grade and used as received. Cell culture medium and reagents were purchased from Gibco laboratories (USA). BD OptEIA ELISA kits tumour necrosis factor- $\alpha$ and interleukin-6 (BD Biosciences, USA). LPS from Escherichia coli and Griess reagent were purchased from Sigma-Aldrich (USA).

Animals. Adult male Sprague-Dawley rats (200-250g; Charles River, St. Constant, QC, Canada) were used. The Animal Ethics Review Committee of the Western University approved the study (Protocol No: 2009-070).

\section{Methods}

Preparation of the aqueous (AQ) and polysaccharide (PS) ginseng extracts. Dried ginseng root samples were shipped to Naturex (USA) for extraction. Samples were ground between $1 / 4$ and $1 / 2$ inch and used to produce the AQ extract [6]. Briefly, $4 \mathrm{~kg}$ ground ginseng roots were soaked three times during five hours in $16 \mathrm{~L}$ of water solution at $40^{\circ} \mathrm{C}$. After extraction, the solution was filtered at room temperature. The excess solvent was then removed by a rotary evaporator under vacuum at $45^{\circ} \mathrm{C}$. The three pools were combined and concentrated again until the total solids on a dry basis were around $60 \%$. These concentrates were lyophilized with a freeze dryer (Labconco, USA) at $-50^{\circ} \mathrm{C}$ under reduced pressure to produce the AQ ginseng extract in powder form. Yield of the powder extracts from the 
concentrates was about $66 \%$. The yields of the final extract (mean \pm standard deviation of $\%$ extractive) from the initial ground root were $41.74 \pm 4.92$.

A solution of AQ extract in distilled water $(10 \mathrm{~g} / 10 \mathrm{~mL})$ was prepared, and the crude PS was precipitated by the addition of four volumes of $95 \%$ ethanol. The PS fraction was collected by centrifugation at 350×g (Beckman Model TJ-6, USA) for 10 minutes and lyophilized to produce the crude PS extract.

To prepare the water soluble polysaccharide extract (WSPE), ginseng roots (500 g) were extracted with 7.0 L of MilliQ (EMD Millipore) water at $100{ }^{\circ} \mathrm{C}$ for $4 \mathrm{~h}$ and filtered through sheets of glass fiber. The solid material was extracted twice under identical conditions. The filtrates were combined, then centrifuged to remove water insoluble materials and supernatants were concentrated $(1.0 \mathrm{~L})$ and precipitated by the addition of $95 \%$ ethanol $(4$ to 1 volumes). After centrifugation, the precipitate was washed and dried by solvent exchange, first using 95\% ethanol, and then absolute ethanol. Crude water soluble polysaccharide extract (WSPE) was obtained with a $20.0 \%$ yield (relative to dry weight of plant material) (Figure 1).

\subsection{Chromatography of ginseng extracts}

High performance liquid chromatography (HPLC) analysis for ginsenoside determination

HPLC analysis on the composition of ginsenosides in the AQ extracts $(100 \mathrm{mg} / \mathrm{ml}$ methanol) was performed with a Waters 1525 HPLC System with a binary pump and UV detector [6]. A reversed-phase Inspire C18 column $(100 \mathrm{~mm} \times 4.6 \mathrm{~mm}$, i.d. $5 \mu \mathrm{m})$ purchased from Dikma Technologies (USA) was used for all chromatographic separations. Gradient elution consisted of [A] water and [B] acetonitrile at a flow of $1.3 \mathrm{~mL} / \mathrm{min}$ as follows: $0 \mathrm{~min}, 80-20 \%$; 0-60min, $58-42 \%$; $60-70 \mathrm{~min}, 10-90 \% ; 70-80 \mathrm{~min}, 80-20 \%$. Absorbance of the eluates was monitored at $203 \mathrm{~nm}$.

\section{Sephadex G-75 chromatography}

AQ ginseng extract $(500 \mathrm{mg}$ ) was dissolved in $5 \mathrm{~mL}$ distilled water and then fractionated by loading to a calibrated Sephadex G-75 column $(47 \times 2.5 \mathrm{~cm})$ equilibrated and eluted with distilled water mobile phase at $4^{\circ} \mathrm{C}$ with a flow rate of $1 \mathrm{~mL} / \mathrm{min}$ [6]. Absorbance of the eluates was monitored at $230 \mathrm{~nm}$. Fractions were collected and lyophilized for the study of bioactivity distribution.

Preparation of the de-proteinated water soluble polysaccharide extracts (DWSPE)

WSPE (50 g) were re-dissolved in $1.5 \mathrm{~L}$ of MilliQ water and partitioned five times with Sevag reagent (1:4 n-butanol:chloroform, v/v, $500 \mathrm{~mL}$ each) to remove proteins [8]. Polysaccharides were precipitated again by ethanol and dried by solvent exchange. This procedure yielded $46.0 \mathrm{~g}$ of the de-proteinated water soluble polysaccharide fraction (DWSPE). The procedure for the preparation of DWSPE from P. quinquefolius is shown in Figure 1. 


\section{Total fractionation of the DWSPE by ion exchange chromatography on DEAE-Cellulose}

DWSPE fraction (20 g) was dissolved in MilliQ water $(200 \mathrm{~mL})$ and loaded on a DEAECellulose column $\left(10.0 \times 20 \mathrm{~cm}, \mathrm{Cl}^{-}\right)$pre-equilibrated with MilliQ water. The column was eluted first with $4.0 \mathrm{~L}$ of MilliQ water at a flow rate of $10 \mathrm{~mL} / \mathrm{min}$ ( 4 bar column pressure) to obtain the unbound or neutral fraction (DWSPE-N) and then with $4.0 \mathrm{~L}$ of $0.5 \mathrm{M} \mathrm{NaCl}$ to obtain the bound or acidic fraction (DWSPE-A). The fractions were concentrated, dialyzed (cut off pore size of $2 \mathrm{KDa}$ ) against MilliQ water and freeze dried to give $15.0 \mathrm{~g}(13.8 \%)$ of the DWSPE-N and $0.9 \mathrm{~g}(0.83 \%)$ of the acidic fraction DWSPE-A. DWSPE-A (0.6 g) was dissolved in $50 \mathrm{~mL}$ MilliQ water and loaded on a DEAE-Cellulose column $\left(10 \times 20 \mathrm{~cm}, \mathrm{Cl}^{-}\right)$. The column was eluted by a stepwise gradient with $2.0 \mathrm{~L}$ of $\mathrm{NaCl}$ aqueous solutions (0.0, $0.1,0.2,0.3$ and $0.5 \mathrm{M}$ each) at a flow rate of $10 \mathrm{~mL} / \mathrm{min}$ (4 bar column pressure). A total of 120 eluate fractions were collected (50 mL each), dialyzed and lyophilized (Figure 2).

High performance gel permeation chromatography-evaporative light scattering detection (HPGPCELSD) analysis

HPGPC was carried out at $40^{\circ} \mathrm{C}$ using a TSK-gel G-3000PWXL column $(7.8$ X $300 \mathrm{~mm}$, $\mathrm{TOSOH}$, Japan) connected to a HPLC system coupled with Diode Array and Evaporative Light Scattering Detectors (DAD-ELSD). The column was calibrated with standard dextrans (5 to 410 KDa range, Figure 7-B). Ten microliters of $20 \mathrm{mg} / \mathrm{mL}$ solutions of DWSPE, DWSPE$\mathrm{N}$ and DWSPE-A were separately injected and eluted with HPLC grade water at a flow rate of $0.8 \mathrm{~mL} / \mathrm{min}$ and monitored using ELSD with a temperature setting at $80^{\circ} \mathrm{C}$.

High performance gel permeation chromatography-multi-detector analysis

AQ and PS ginseng extracts were analysed at $40^{\circ} \mathrm{C}$ with TSK-gel PWXL G-4000PWXL column (7.8 X $300 \mathrm{~mm}$, TOSOH, USA) connected to a Viscotek (Varian Instruments, USA) gel permeation chromatography system with Omnisec software (version 4.5, Viscotek, USA) for data acquisition. Solutions of AQ and PS extract $(1 \mathrm{mg} / \mathrm{mL})$ were filtered with $0.2 \mu \mathrm{m}$ nylon filter and used for analysis. Each sample $(100 \mu \mathrm{l})$ was injected and eluted with $0.3 \mathrm{M}$ sodium chloride $(\mathrm{NaCl})$ mobile phase at a flow rate of $1 \mathrm{~mL} / \mathrm{min}$ and monitored using a multiple detectors system for light scattering, refractive index and viscosity. Pullulan polysaccharide reference standard was analyzed as a positive control.

\subsection{Analysis of the monosaccharides composition in WSPE by HPLC-ELSD}

Carbohydrate analysis represents a challenge in analytical chemistry since neutral or acidic saccharides (mono, oligo and poly) have little UV activity. In our study, evaporative light scattering detection (ELSD) was used. The ELSD does not require the solutes of interest to have any optical properties; and the only requirement is that the eluent be more volatile than the solutes. WSPE $(20 \mathrm{mg})$ was dissolved in $10 \mathrm{~mL}$ of $2 \mathrm{~N} \mathrm{HCl}$ solution and was boiled for $2 \mathrm{~h}$. The hydrolyzed product was neutralized ( $\mathrm{pH} \mathrm{6-7)}$ and centrifuged before analysis. 


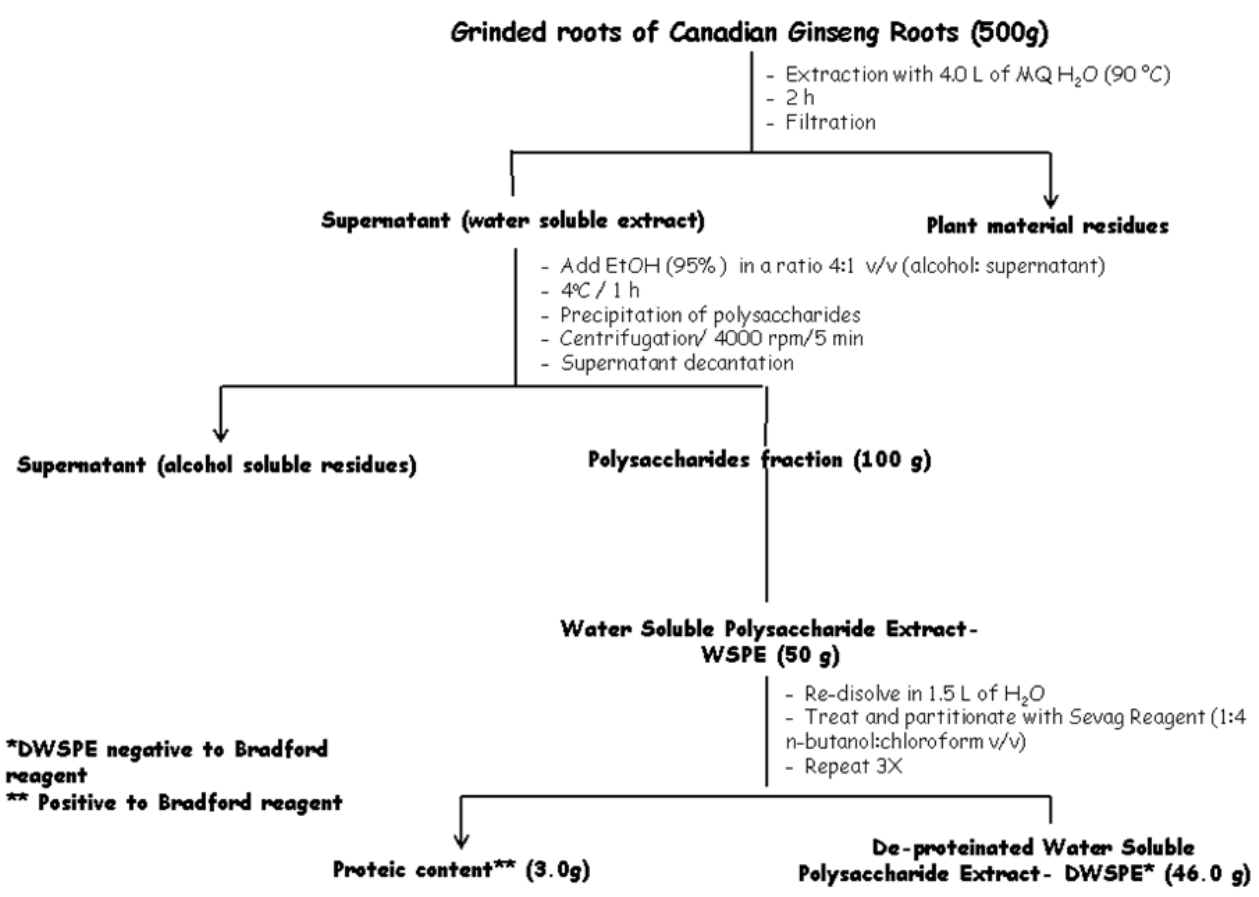

Figure 1. Preparation of the water soluble polysaccharides extract (WSPE) and the de-proteinated extract from the roots of 4-year-old Ontario-grown Panax quinquefolius.

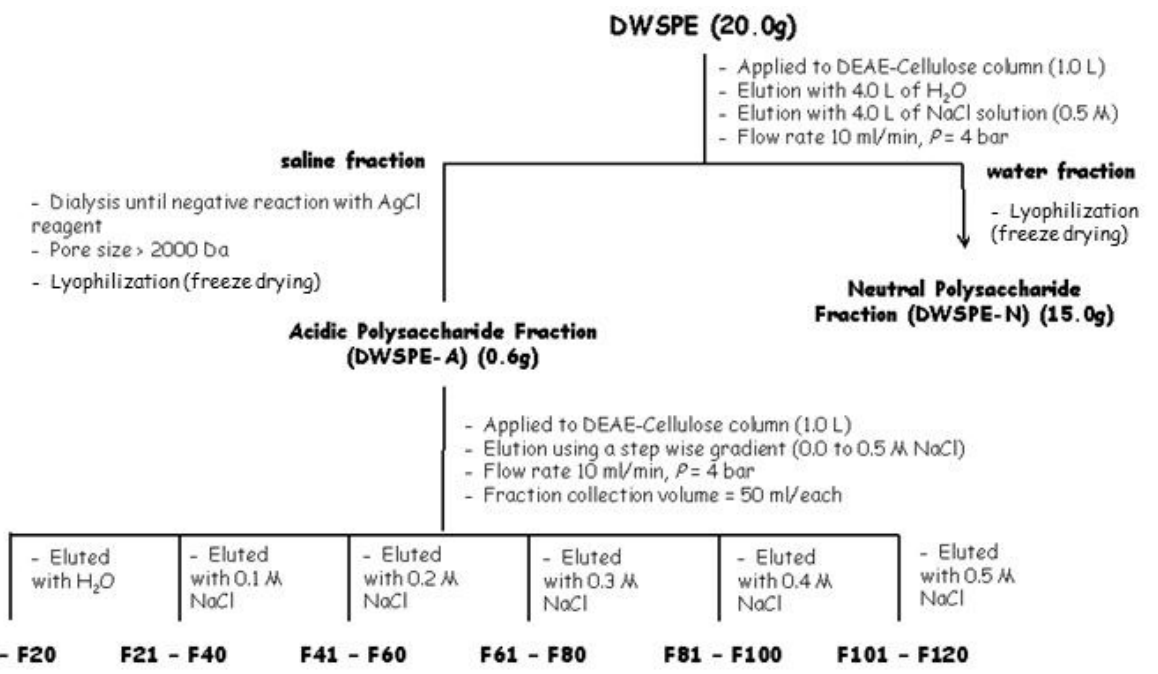

Figure 2. Step wise fractionation of the de-proteinated water soluble ginseng polysaccharide extract (DWSPE). 
HPLC analysis was conducted using an 1100 series HPLC-DAD-ELSD system (Agilent Technologies Inc., Santa Clara, CA, USA). To enhance resolution of monosaccharides, analysis was performed using two separate columns (Figure 2). Glucose, galactose, arabinose, mannose and xylose were eluted using a Rezex RPM Monosaccharide PB+2 (8\%) (Phenomenex, Torrance, California) column with a mobile phase of $100 \%$ water (Chromasolv Plus, HPLC grade) isocratically at $80{ }^{\circ} \mathrm{C}$ and a flow rate of $0.6 \mathrm{~mL} / \mathrm{min}$. The ELSD temperature was set to $80^{\circ} \mathrm{C}$. Galacturonic acid and rhamnose content were examined using a Luna $5 \mu \mathrm{NH}_{2} 100 \AA$ column (Phenomenex, Torrance, California) and eluted with a mobile phase of acetonitrile and water $(80: 20)$ at $40{ }^{\circ} \mathrm{C}$ and a flow rate of $3 \mathrm{~mL} / \mathrm{min}$. The ELSD temperature was set to $44^{\circ} \mathrm{C}$.

\subsection{Pharmacological evaluation}

\subsubsection{In vivo study}

Adult male rats (250-300 gm) were treated with $125 \mathrm{mg} / \mathrm{kg}$ of AQ extract or crude PS fraction dissolved in saline by gastric garvage $(10 \mathrm{ml} / \mathrm{Kg}$ bwt $)$ once daily for 3 or 6 consecutive days, and examined $24 \mathrm{hr}$ after the last dose. Animals were anesthetized with i.m. injection ( 80 and $5 \mathrm{mg} / \mathrm{kg}$ b.wt. ketamine and xylazine, respectively) and the trachea was cannulated for lung bronchoalveolar lavage (BAL) with Dulbecco's phosphate-buffered saline (PBS) to collect alveolar macrophages.

Blood was collected into heparinized tubes from rats by intracardiac puncture, samples were immediately centrifuged at $3000 \mathrm{rpm}$ for 10 minutes and the plasma was separated, aliquoted and stored at $-20^{\circ} \mathrm{C}$ until use.

\subsubsection{In vitro study}

\section{Cell culture}

Rat alveolar macrophages were collected by BAL using cannulated 10-ml syringe with three $10 \mathrm{ml}$ washes of PBS. Fluid recovered from BAL was centrifuged at $1000 \mathrm{rpm}$ for 5 minutes. Cells were cultured in RPMI-1640 medium supplemented with 10\% Fetal Bovine Serum (FBS), 25mM HEPES, $2 \mathrm{mM}$ Glutamine, $100 \mathrm{IU} / \mathrm{ml}$ penicillin and $100 \mu \mathrm{g} / \mathrm{ml}$ streptomycin in 96-well tissue culture plates, at a density of $2 \times 10^{5}$ cells per well at $37^{\circ} \mathrm{C}$ maintained in a humidified incubator with $5 \% \mathrm{CO}_{2}$.

\section{Cell treatment}

\section{Immuno-stimulatory effect}

Experiments to evaluate dose-related stimulation of inflammatory mediators profile in vitro were carried out by treating and incubating rat alveolar macrophages with 0, 50, 100 and $200 \mu \mathrm{g} / \mathrm{ml}$ of ginseng extracts for 24 hours and washed before challenging with LPS $(1 \mu \mathrm{g} / \mathrm{mL})$ was used as positive control. The 24 hours-production of NO, TNF- $\alpha$ and IL-6 in culture medium was determined. 


\section{LPS-induced immuno-suppression}

To examine the direct inhibitory effect of ginseng extracts on LPS-stimulated immune function, we pre-treated the macrophages with $0,50,100$ or $200 \mu \mathrm{g} / \mathrm{ml}$ of ginseng extracts for 24 hours and washed before challenging with LPS $(1 \mu \mathrm{g} / \mathrm{ml})$. The 24-hour cytokine production induced by LPS was determined by measuring NO, TNF- $\alpha$ and IL-6 levels in the culture medium.

\subsection{Quantification of NO, TNF- $\alpha$ and IL-6}

TNF- $\alpha$ and IL-6 concentrations in supernatants from cultured cells and plasma were analyzed with ELISA [6]. Samples were evaluated with rat cytokine-specific BD OptEIA ELISA kits (BD Biosciences, USA) according to the manufacturer's protocol. NO production was analyzed as accumulation of nitrite in the culture medium. Nitrite in culture supernatants was determined with Griess reagent (Sigma-Aldrich, USA) as previously described [6].

\subsection{Statistical analysis}

In vivo and in vitro experiments were performed at least three separate times. All statistical analyses were performed with GraphPad prism 4.0a Software (GraphPad Software Inc., USA). Data were presented as the mean \pm standard deviation (SD) of triplicates from three independent experiments. Data sets with multiple comparisons were evaluated by one-way analysis of variance (ANOVA) with Dunnett's post-hoc test. $P<0.05$ was considered to be statistically significant.

\section{Results}

\subsection{Phytochemistry}

Ginsenoside Compositon. AQ extract contained a total ginsenoside content of $13.87 \%$ dry weight of extract and showed characteristics of Panax quinquefolius with $\mathrm{Rb} 1$ and Re as the predominant ginsenosides, with no detectable Rf and minimal levels of Rg1 (Fig.3).

Crude PS extract. The yield of crude PS fraction by four volumes of $95 \%$ ethanol precipitation was $10 \%$ dry weight of root materials. A representative G-75 chromatographic profile of the crude PS extract is shown in Figure 4 [6]. The major PS peak (with a elution volume of 100 ML) had an estimated average molecular weight of $73 \mathrm{kDa}$, while there were two minor, less well- resolved peaks (Fig 4 ).

Monosaccharide composition of the WSPE determined by HPLC-ELSD. Representative chromatograms showing individual monosaccharides in water soluble polysaccharide extract (WSPE) are shown in Figure 5 and 6. Glucose was found to be the major neutral monosaccharide present in WSPE with amounts ranging from 77 to $86 \%(w / w)$. Galactose and arabinose were present in similar amounts with galactose being present at levels of 6.8 
to $7.5 \%(\mathrm{w} / \mathrm{w})$ and arabinose being present at levels between 4.5 to $5.9 \%(\mathrm{w} / \mathrm{w})$. Galacturonic acid was also identified on WSPE at levels ranging from 8.7 to $9.5 \%(\mathrm{w} / \mathrm{w})$. Mannose and xylose were also monitored but were not detected in the sample.

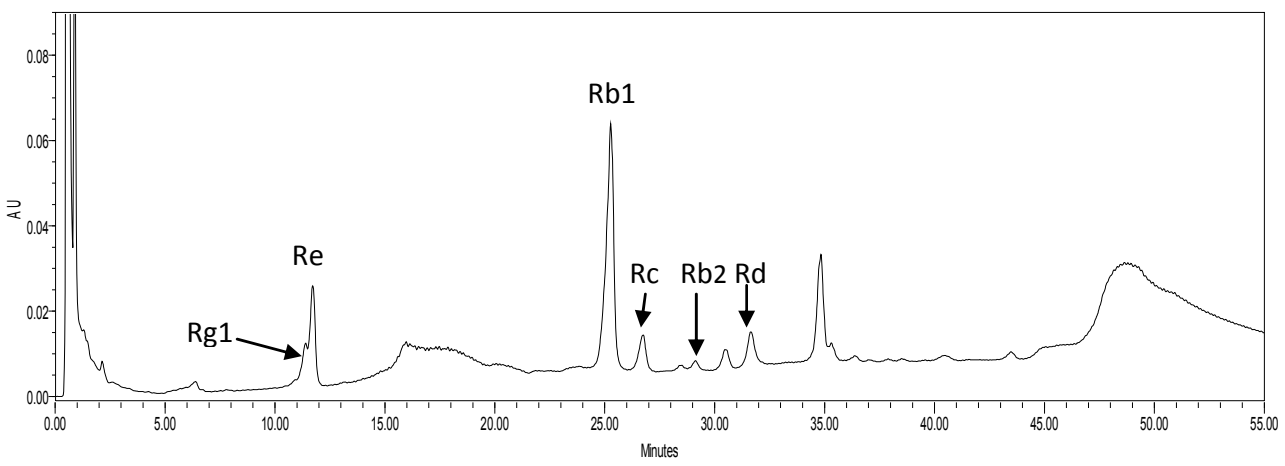

Figure 3. HPLC ginsenoside profile of Ontario-grown American ginseng aqueous extract. A reversedphase Inspire C18 column was used with gradient elution consisted of [A] water and [B] acetonitrile: 0min, 80-20\%; 0-60min, 58-42\%; 60-70min, 10-90\%; 70-80min, 80-20\%. Absorbance of the eluates was monitored at $203 \mathrm{~nm}$.

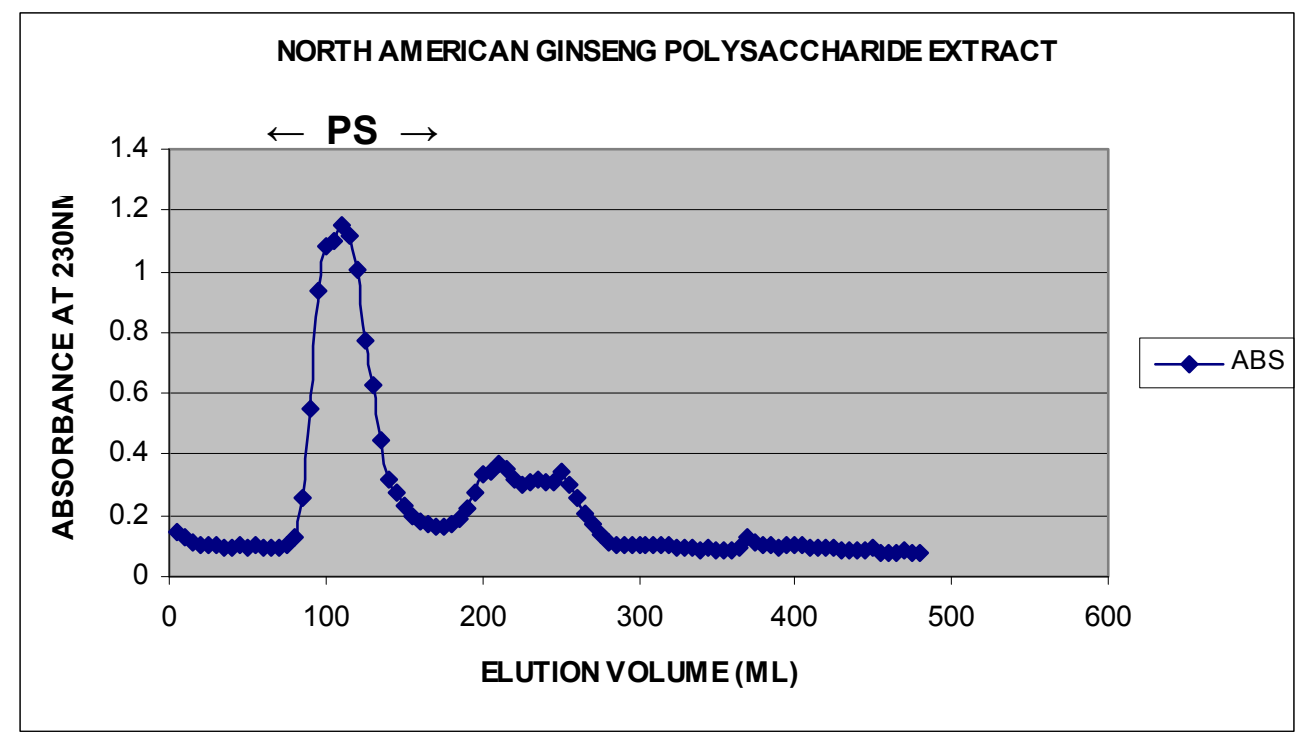

Figure 4. Sephadex G-75 $(47 \times 2.5 \mathrm{~cm})$ chromatographic fractionation of PS extracts of ginseng. A calibrated column was loaded with $500 \mathrm{mg}$ of the extract, and then eluted with distilled water at a flow rate of $1 \mathrm{~mL} / \mathrm{min}$. The $\mathrm{y}$-axis is the absorbance at $230 \mathrm{~nm}$ while the $\mathrm{x}$-axis represents the elution volume $(\mathrm{mL})$.

Total fractionation of the DWSPE by ion exchange chromatography on DEAE-Cellulose. To further analyse the WSPE, the material was de-proteinated using the Sevag method, giving a 
DWSPE with a yield of $92 \%$ and the protein content yield of $1.8 \%$ (relative to the dry weight of WSPE). Fractionation of WSPE by a combination of anion-exchange on DEAE-cellulose and gel permeation chromatographies with a procedure shown in Figure 1 revealed the elution of a neutral fraction with water (N-DWSPE, $75 \%$ relative to the dry weight of DWSPE) and an acidic fraction (A-DWSPE, 4.5\%) with a $0.5 \mathrm{M} \mathrm{NaCl}$ solution (Figure 7). Both fractions presented a wide and complex molecular weight distribution ranging from 5 to $410 \mathrm{KDa}$. The acidic fraction A-DWSPE containing most likely uronic acids was subjected to a second fractionation by DEAE-cellulose chromatography.

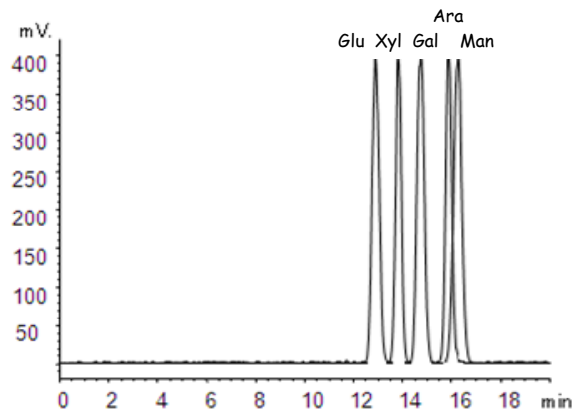

V

(A)

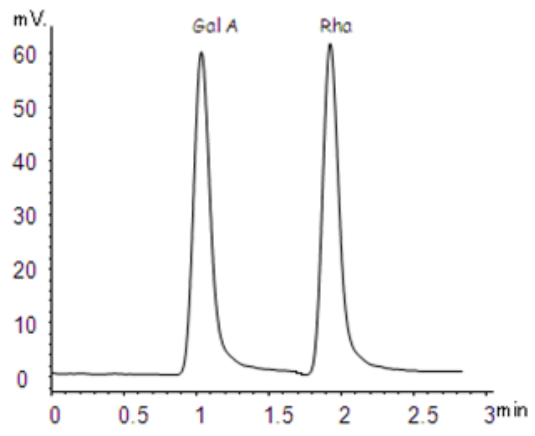

(B)

Figure 5. Representative chromatograms of the novel analytical method by HPLC-ELSD for the analysis of seven monosaccharides including glucose (Glu), galactose (Gal), arabinose (Ara), Xylose (Xyl), Mannose (Man) (A), galacturonic acid (Gal A) and rhamnose (Rha) (B).
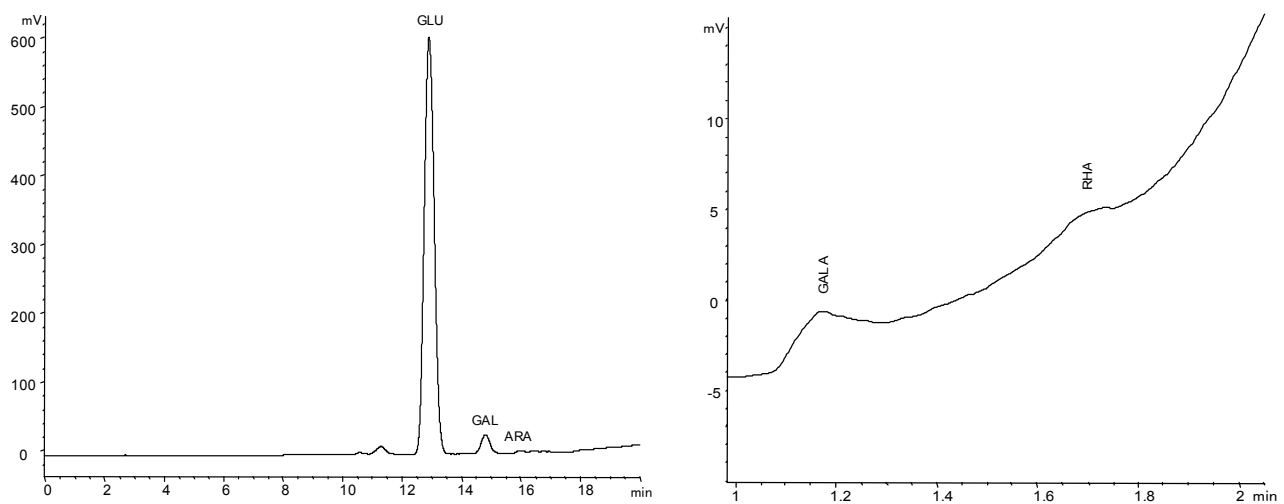

Figure 6. Representative HPLC-ELSD Chromatograms of monosaccharide detected in WSPE, including glucose (Glu), galactose (Gal), arabinose (Ara) (A), galacturonic acid (Gal A) and rhamnose (Rha) (B).

Secondary Fractionation with the A-DWSPE on DEAE-Cellulose column chromatography. The elution of $0.6 \mathrm{~g}$ of A-DWSPE on a DEAE-cellulose chromatography was carried out using a stepwise gradient of $\mathrm{NaCl}$. With these elution steps, A-DWSPE was separated into six 
fractions (Figure 2): A-DWSPE-1 (F1-F20, 96 mg, 16 \%), A-DWSPE-2 (F21-F40, 54mg, 9\%), ADWSPE-3 (F41-F60, 162 mg, 27\%), A-DWSPE-4 (F61-F80, 120mg, 20\%), A-DWSPE-5 (81F100, 78mg, 13\%) and A-DWSPE-6 (F101-F120, 60mg, 10\%) corresponding to the elution with $0.0,0.1,0.2,0.3,0.4$ and $0.5 \mathrm{M} \mathrm{NaCl}$ solutions, respectively.

(A)

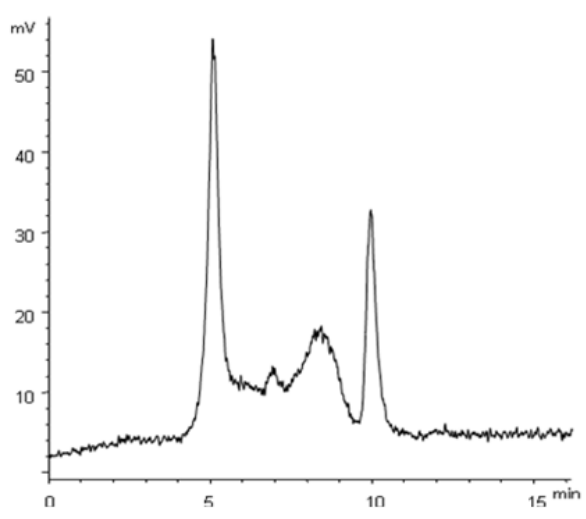

(C)

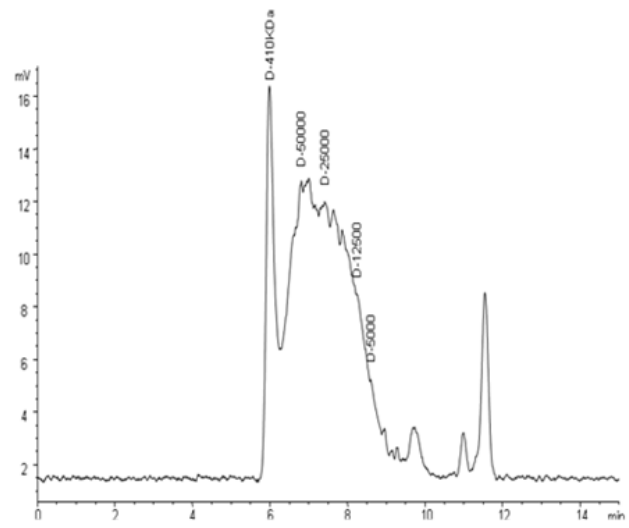

(B)

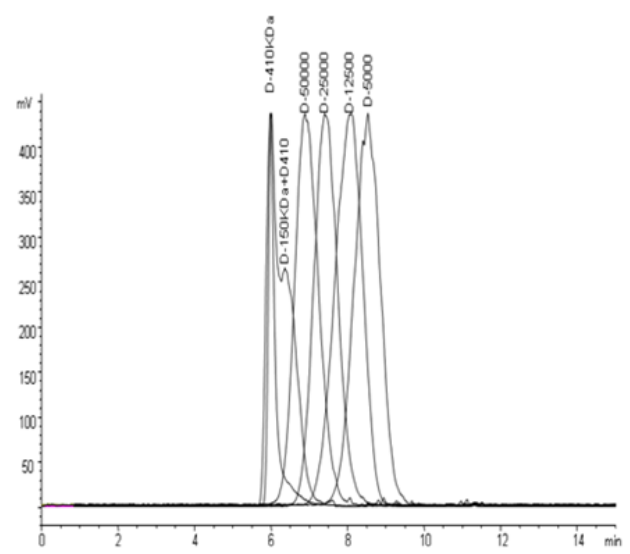

(D)

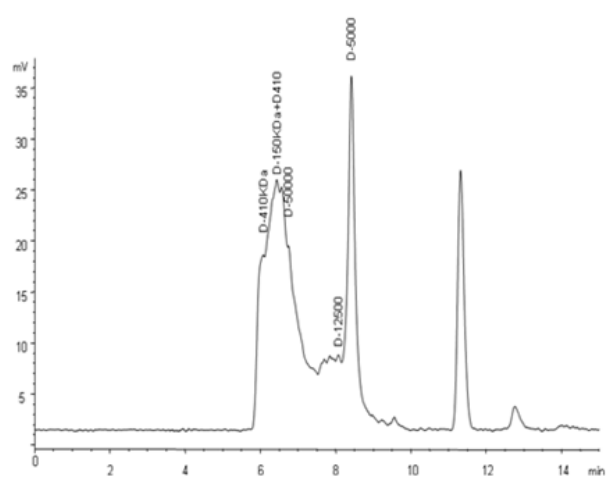

Figure 7. Representative chromatrograms by HPGPC-ELSD analysis; (A) DWSPE sample, (B) Dextran standards mixture, (C) DWSPE-N and (D) DWSPE-A. All samples were eluted under the same conditions.

In order to further analyze the unique NA ginseng polysaccharide samples, GPC with multiple detectors was utilized. Figure 8 shows the results of multiple pullulan polysaccharide standards ranging from $1800 \mathrm{Da}$ to 1,050,000 Da. The utilized column gave 
good resolution of the individual standards over this range. Figure 8 shows the results of the multi-detector system containing 4 different detectors, i.e. refractive index (RI), right angle light scattering, low angle light scattering and viscometer. In Figure 9, the crude polysacchride extract is observed to have three major peaks with $\mathrm{M}_{\mathrm{w}}$ values of $1092 \mathrm{kDa}, 135$ $\mathrm{kDa}$ and $12 \mathrm{kDa}$ (Table 1). The major peak at $1092 \mathrm{kDa}$ accounts for $66 \%$ of the weight fraction. Figure 9 shows the deproteinated polysaccharide fraction, in which the $\mathrm{M}_{\mathrm{w}}$ values are largely unchanged (Table 1). However, the protein content fraction (PTF) contains only a small amount of carbohydrates with the majority of the high molecular weight fraction removed. The acid fraction contains 3 peaks, of generally lower $\mathrm{M}_{\mathrm{w}}$ values, while the neutral fraction obtained from anionic exchange of DWSPE contains only one broad peak (Figure 9).
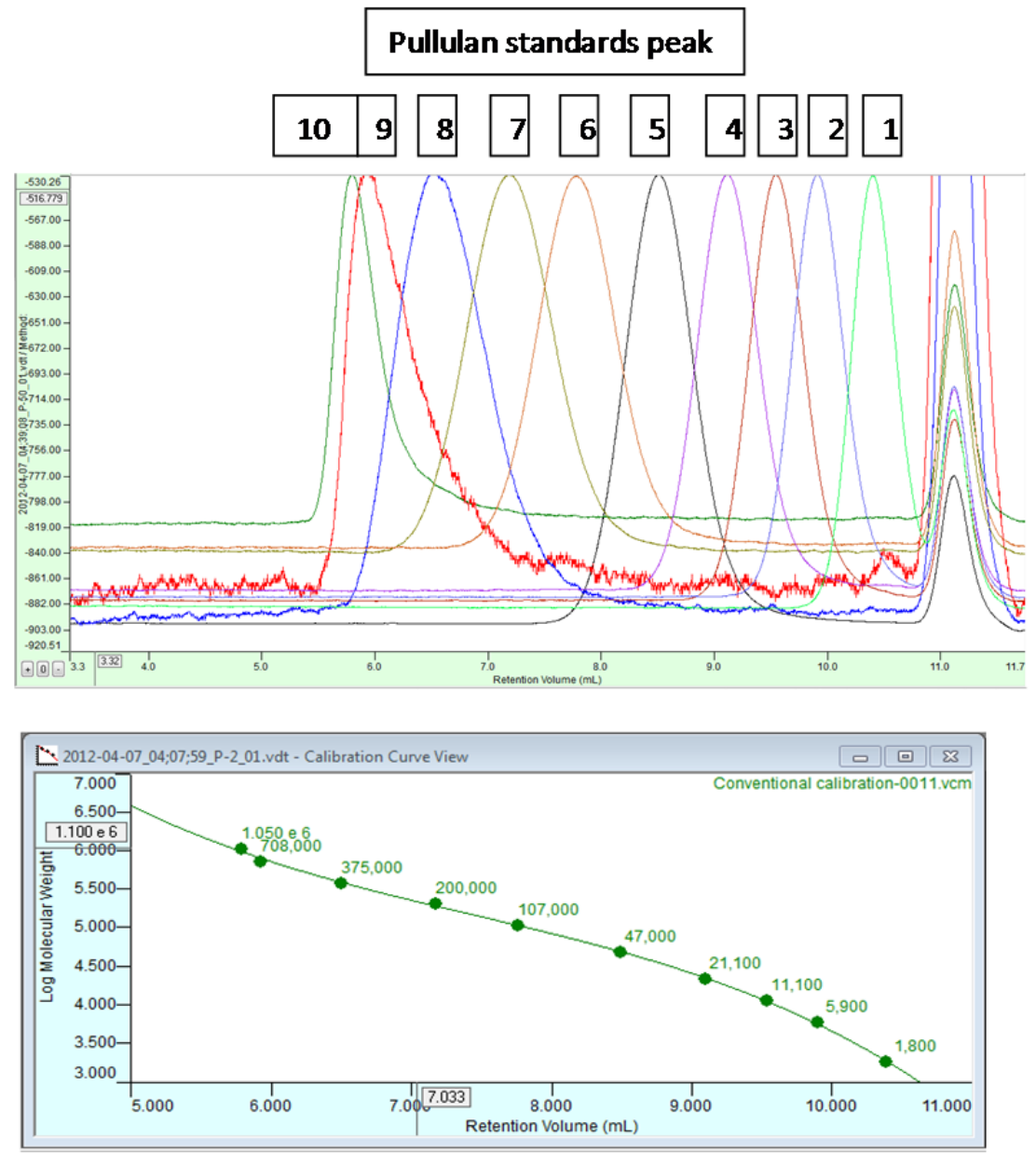

Figure 8. a) Resolution of individual pullulan standards: peak 1, 1800; peak 2, 5900; peak 3, 11,100; peak 4, 21,100; peak 5, 47,000; peak 6, 107,000; peak 8, 375,000; peak 9, 708,000; peak 10, 1,050,000 and b) calibration curve of pullulan standards through G-4000PWXL column. 

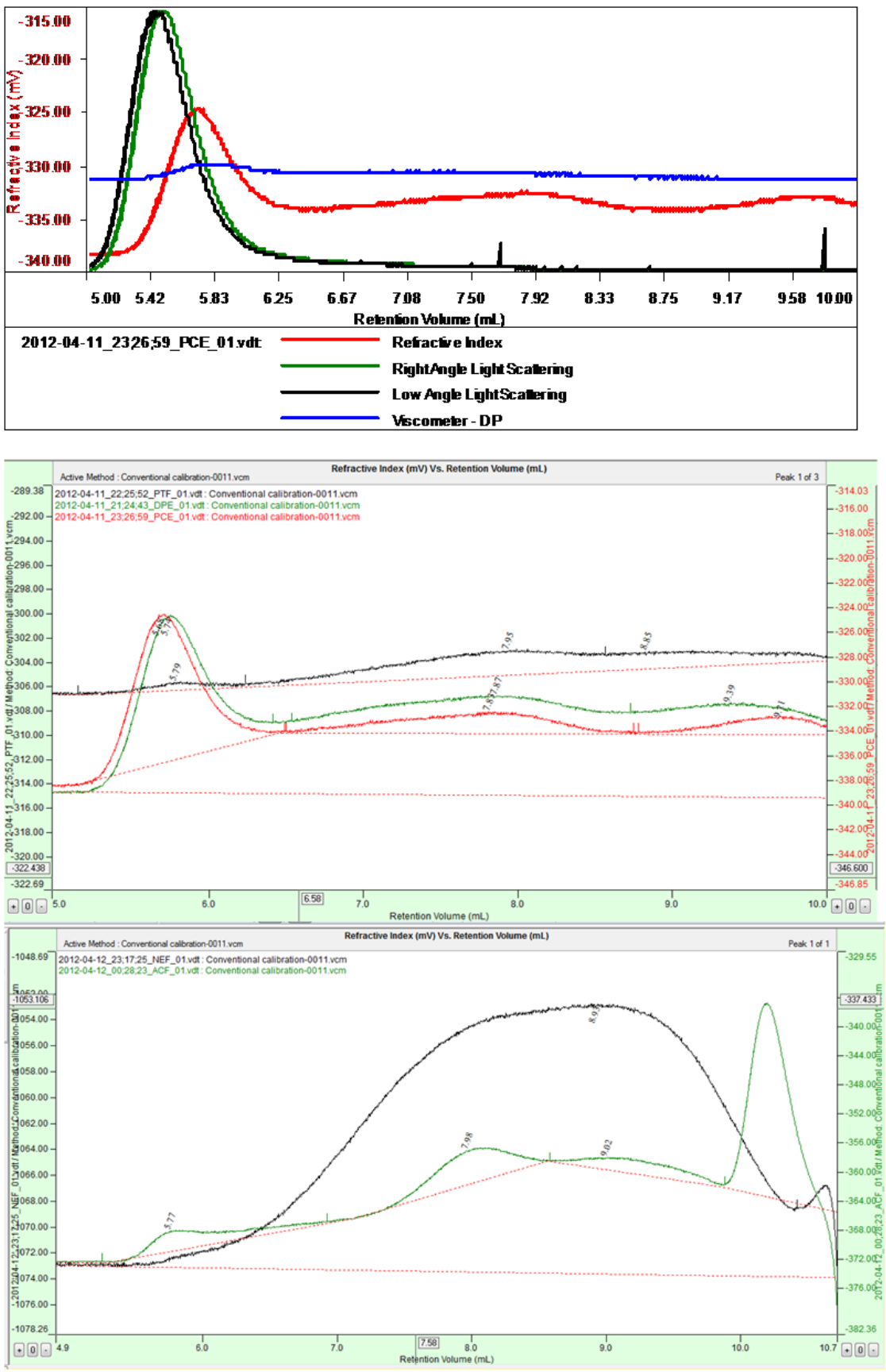

Figure 9. a) GPC traces showing traces from the individual detectors of the PCE (Crude extract of polysaccharides), b) RI plot for the PCE (red), DWSPE (green) and PTF (black) samples. The high 
molecular weight peak at retention volume $5.79 \mathrm{~mL}$ has very low intensity in the PTF sample, which shows that this sample has a very low content of polysaccharide., c) RI plot of the neutral extract (black) and the acidic extract (green) from the ion exchange column, the neutral extract shows much bigger RI area, therefore much higher sample recovery.

\begin{tabular}{|l|l|l|l|}
\hline PCE & 1 & 2 & 3 \\
\hline Mn (Dalton) & 995,589 & 106,001 & 9,502 \\
\hline Mw(Dalton) & $1,092,000$ & 135,019 & 12,037 \\
\hline $\mathrm{Mz}$ (Dalton) & $1,188,000$ & 172,473 & 15,433 \\
\hline RI area (mVmL) & 5.84 & 2.03 & 1.03 \\
\hline $\begin{array}{l}\text { Weight fraction } \\
\text { (\% of RI area) }\end{array}$ & $65.6 \%$ & $22.8 \%$ & $11.6 \%$ \\
\hline
\end{tabular}

Table 1. Molecular weight data of the PCE Extract.

\section{Immunomodulatory activity}

In vivo effect. Treatment with AQ extract for 3-6 days produced marked stimulation of alveolar macrophages as determined by increased production of NO and TNF- and IL-6 following culturing for $24 \mathrm{hrs}$, reaching activities that were $50-100 \%$ of the positive (LPS) control (Figure 10). This immunostimulatory effect was also reflected in the elevation of plasma TNF- and IL-6 levels of treated animals (Figure 11). However, the responsiveness of macrophages collected from ginseng treated animals to LPS stimulation ex vivo showed $>50 \%$ to $100 \%$ reduction in NO, TNF- $\alpha$ and IL- 6 production as compared to those non-ginseng treated controls, especially with 6 days of ginseng treatment (Figure 10). These data showed that orally administered AQ extract had both immuno-stimulatory and anti-inflammatory effect. Data presented in Figure 12 showed that this immunomodulatory activity could be extended to the PS extract on the basis of its effect on macrophage NO production and the LPS responsiveness.

(i) NO

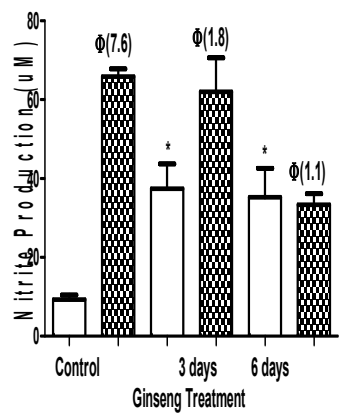

(ii) TNF- $\alpha$

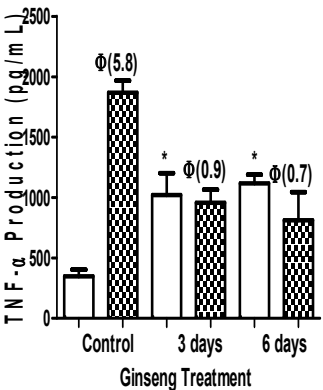

(iii) IL-6

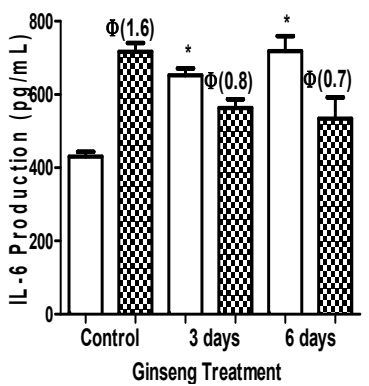

Figure 10. Figure 10. Orally administered ginseng AQ extract: elevated cytokine production and reduced LPS-stimulatd cytokine production in cultured alveolar macrophages. Alveolar macrophages of rats treated orally with 0 and $125 \mathrm{mg} / \mathrm{kg}$ ginseng AQ extract for 3 and 6 Days were cultured for 24 hours to measure production of $\mathrm{NO}$ and cytokines (by ELISA) $\square$. To determine responsiveness to 
LPS stimulation, ginseng treated macrophages were exposed to $1 \mathrm{ug} / \mathrm{ml}$ LPS in culture to determine changes in $24 \mathrm{hr} \mathrm{NO}$ and cytokine production $\mathbf{m i n}$. Three independent experiments were performed and the data were shown as mean \pm SD. Datasets were evaluated by ANOVA. ${ }^{*}$ Values $\mathrm{P}<0.05$ compared to the untreated control were statistically significant. $\Phi$ values in bracket denote fold increase in LPS-stimulated cytokine production over control.

(i) TNF- $\alpha$

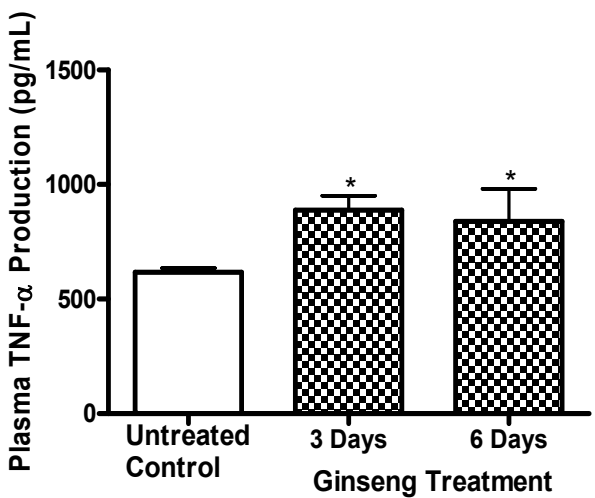

(ii) IL-6

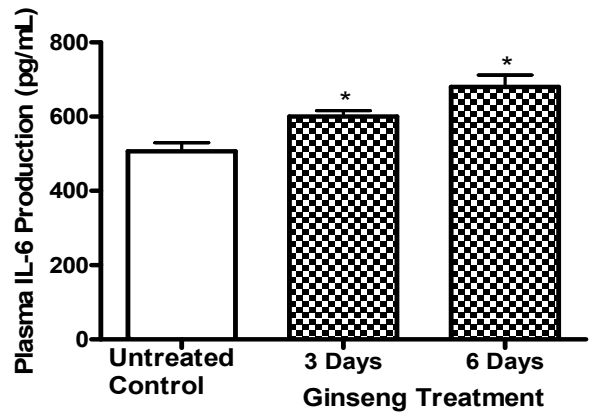

Figure 11. Ginseng AQ extract treatment elevated plasma (i) TNF- $\alpha$ and (ii) IL-6 levels. Rats were treated orally with $125 \mathrm{mg} / \mathrm{kg}$ ginseng AQ extract for 3 and 6 days $\mathbf{m i n}$. Plasma cytokine concentrations were determined by ELISA. Three independent experiments were performed and the data were shown as mean \pm SD. Datasets were evaluated by ANOVA. ${ }^{*}$ Values $P<0.05$ compared to the untreated control were statistically significant.

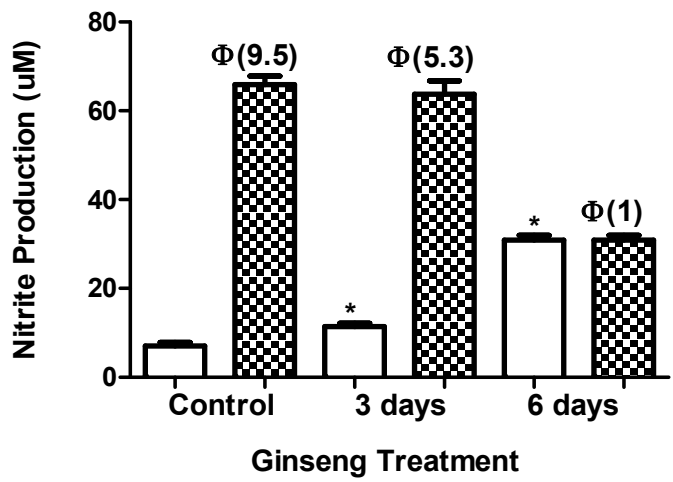

Figure 12. Orally administered ginseng PS extract ( $125 \mathrm{mg} / \mathrm{kg})$ up-regulated NO production $\square$ and reduced responsiveness to LPS (LPS $1 \mu \mathrm{g} / \mathrm{ml}$ ) stimulation $\mathbf{m i n}$ in cultured alveolar macrophages. Cells from untreated controls were treated with LPS $1 \mu \mathrm{g} / \mathrm{ml}$ as positive control for macrophage responsiveness. NO was determined by Griess reaction assay. Three independent experiments were performed and the data were shown as mean \pm SD. Datasets were evaluated by ANOVA. ${ }^{*}$ Values $\mathrm{P}<0.05$ compared to the untreated control were statistically significant. $\Phi$ values in bracket denote fold increase in LPS-stimulated NO production over control. 
(a) NO

\section{(i) Aqueous (AQ) extract}

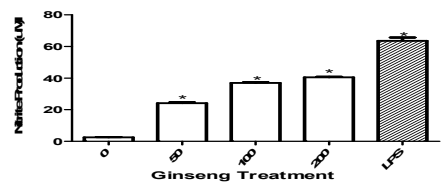

(ii) Crude Polysaccharide (PS) extract

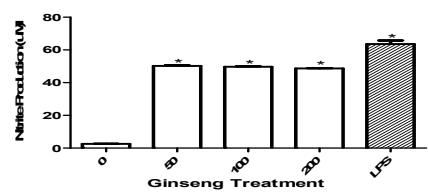

(iii) Acidic PS extract

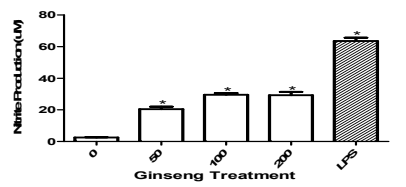

(iv) Neutral PS extract

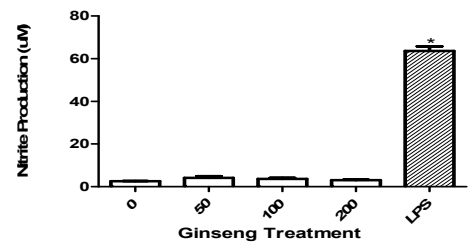

(b) TNF- $\alpha$
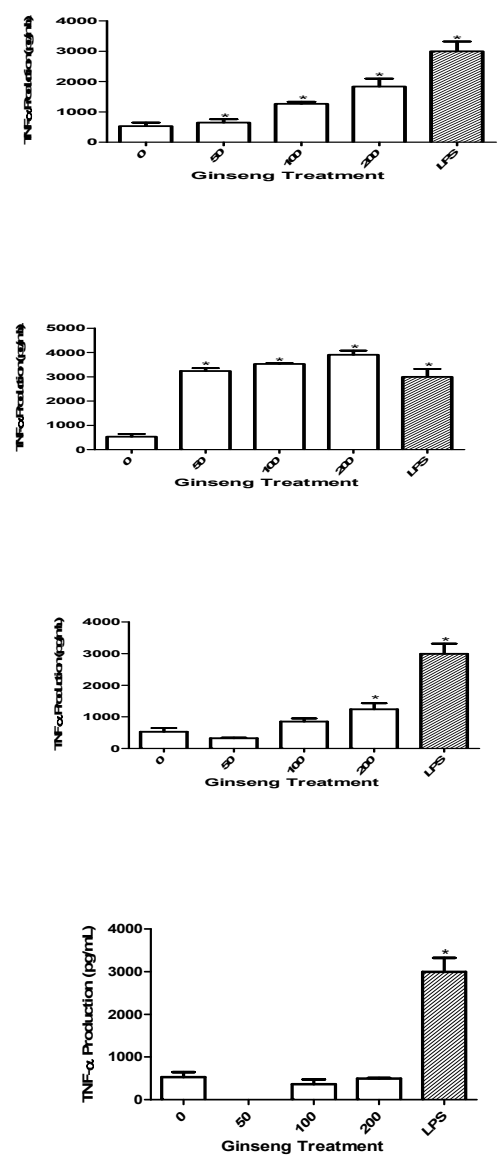

Figure 13. Immuno-stimulatory effects in vitro of AQ, crude PS, acidic PS and neutral PS extracts on 24 hours macrophage production of (a) NO and (b) TNF- $\alpha$. Alveolar macrophages isolated from control rats were treated with $0,50,100$ and $200 \mu \mathrm{g} / \mathrm{ml}$ of ginseng extracts for 24 hours, and the culture supernatants were analysed for NO and TNF- $\alpha$ by Griess reaction assay and ELISA, respectively. Cells treated with LPS $(1 \mu \mathrm{g} / \mathrm{ml})$ were used as positive controls. Three independent experiments were performed and the data were shown as mean \pm SD. Datasets were evaluated by ANOVA. ${ }^{*}$ Values $\mathrm{P}<$ 0.05 compared to the untreated (vehicle) control were statistically significant.

\section{In vitro effect}

Both AQ, crude PS and acidic PS showed stimulation of NO and TNF- $\alpha$ production by alveolar macrophages in vitro (Figure 13). The magnitude of the response to PS was greater than those induced by acidic PS or AQ extract. Neutral PS was devoid of activity. The lack of 
concentration-dependent effect of PS was probably due to its high potency and inducing its maximum effect at the concentration studied. Data presented in Figure 14 showed how pretreatment with various extracts for $24 \mathrm{hrs}$ altered the subsequent response to LPS challenge. Since prior LPS treatment was known to cause desensitization of subsequent response to LPS, this was used as a positive control to evaluate the immunosuppressive effect of ginseng extracts. It was apparent that PS was the most effective in reducing the NO and TNF- $\alpha$ response to LPS, while the AQ and acidic PS extracts were similar, and neutral PS was inactive.

(a) NO

\section{(i) AQ Extract}
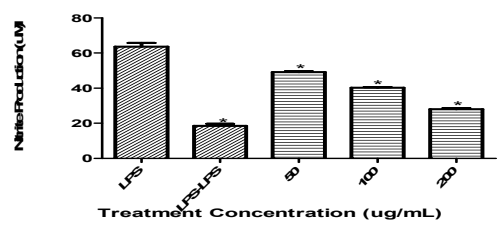

(ii) Crude PS extract

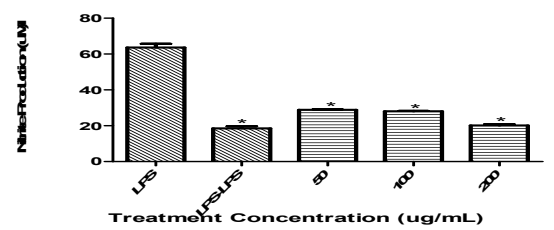

(iii) Acidic PS Extract

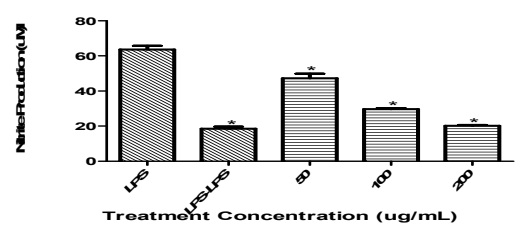

(iv) Neutral PS Extract

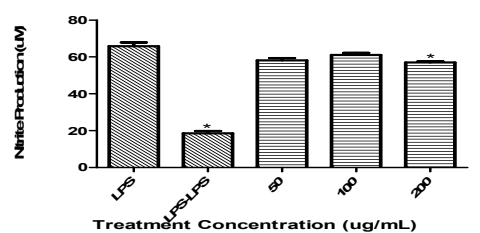

(b) TNF- $\alpha$
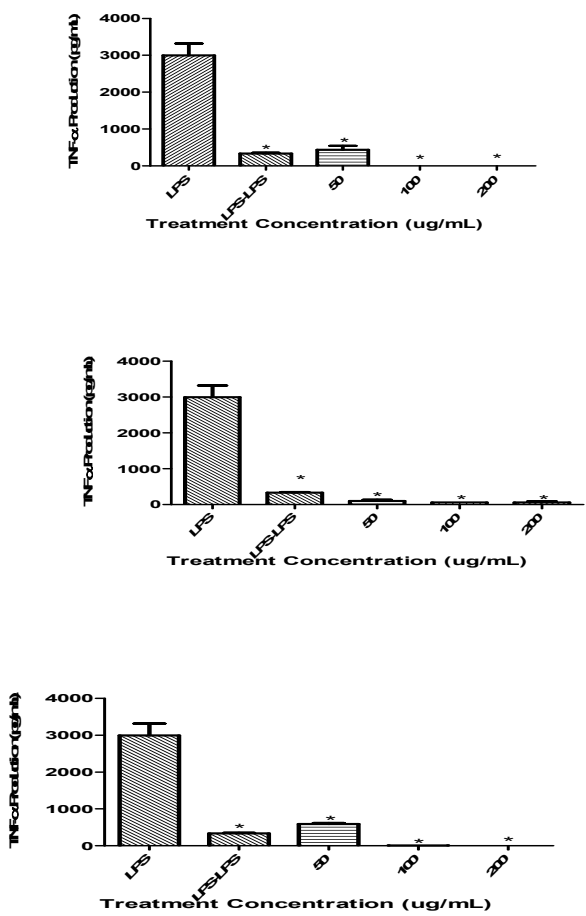

Figure 14. In vitro effects of AQ, crude PS, acidic PS and neutral PS extracts on LPS-stimulated 24 hours macrophage production of (a) NO and (b) TNF- $\alpha$. Rat Alveolar macrophages were pre-treated with 
ginseng extracts $(0,50,200 \mu \mathrm{g} / \mathrm{ml})$ for 24 hours and were washed before challenged with LPS $1 \mu \mathrm{g} / \mathrm{ml}$. $24 \mathrm{hr}$-pretreatment with LPS prior to LPS stimulation was used as positive control to demonstrate desensitization of macrophage responsiveness (LPS-LPS). NO and TNF- $\alpha$ were determined by Griess reaction assay and ELISA, respectively. Three independent experiments were performed and the data were shown as mean \pm SD. Datasets were evaluated by ANOVA. ${ }^{*}$ Values $\mathrm{P}<0.05$ compared to the LPS positive control were statistically significant.

\section{Discussion}

Medicinal plants have been in use for human health for thousands of years, yet polysaccharides have only been recognized recently as a major contributor to the bioactivity of these traditional medicines. Polysaccharides from plant sources with immunomodulatory, anti-tumor, anti-viral, anti-bacterial, anti-inflammatory, anti-oxidant, and anti-diabetogenic activities have been reported [2, 9-15]. And a few polysaccharides, including lentinan, Astragalus polysaccharide, polyporus polysaccharide and Achyranthes bidentata polysaccharide have been licensed for clinical application in China [16]. In Canada, a polysaccharide-enriched American ginseng extract (Cold Fx®) has been licensed in 2007 as a natural health product to 'help reduce the frequency, severity and duration of cold and flu symptoms by boosting the immune system' with an estimated annual sale of over \$48M [4, 5]. Our findings on the paradoxical effects of AQ and PS extract on macrophage function in vivo may have significant implication in the use of American ginseng polysaccharides in several clinical applications.

The structure and biological activities of the polysaccharides from the roots, leaves and fruits of Panax ginseng have recently been reviewed by Sun [2]. There is limited information on American ginseng. Our study in vitro has demonstrated the up-regulation of inflammatory mediators production by AQ and crude PS extracts in rat alveolar macrophages (Figure 13) which validated what we have previously reported [ 3 and 6]. In addition we have demonstrated specificity of PS in that acidic but not neutral species of the PS was bioactive. Following sub-acute oral administration, both PS and AQ extracts were also immuno-stimulatory based on elevation of plasma cytokine levels and increase in the function of alveolar macrophages ex vivo. The immuno-stimulatory dosage used in the present in vivo study was comparable to those proven to be effective for cardiovascular health and for protection against diabetic retinopathy, neuropathy and cardiomyopathy reported by other investigators using identical ginseng extracts and in the same animal species [17-19]. However, the magnitude of the immunostimulatory effect in vivo was smaller than the in vitro response. This may be related to the lower bioavailability of the orally-administered ginseng extract. The pharmacokinetics of oral ginseng PS is not known, but the recovery of major ginsenosides in plasma after oral administration was quite low [20]. Result of our in vitro and in vivo studies was supportive of what was reported for CVTE002 (a patented, poly-furanosyl-pyranosyl polysaccharide-rich extract of the root of North American ginseng): stimulation of normal mouse spleen cells and immunoglobulin $G$ production as well as activation of peritoneal exudate macrophages leading to enhanced cytokine stimulation in treated mice. 
In addition to the well-recognized immuno-stimulatory activity of ginseng, an antiinflammatory effect was shown in the present study as reflected in the reduced responsiveness of alveolar macrophages collected from ginseng-treated animals to LPS challenges ex vivo (Figure 10). This apparent anti-inflammatory effect of ginseng PS is different from what we have previously reported for a specific component(s) of the alcoholic extract of American ginseng as well as the anti-inflammatory effects that have been ascribed to some ginsenosides and their metabolites [6, 21-22]. This potential anti-inflammatory mechanism is being validated by evaluating changes in LPS-induced inflammatory response following polysaccharide pretreatment in vivo in our on-going research. The intent is to determine whether ginseng PS causes desensitization of immune cells as reported for LPS [23]. This action of ginseng, when proven, may be particularly relevant to bacterial infection and related toxicemia. An anti-inflammatory effect of ginseng polysaccharide has been reported by Zhao et al. using a model of auto-immune disease, as evidenced by the reduction in the expression of TNF- $\alpha$ and IFN- $\gamma$ in lymphocytes in the enteric mucosal immune system of rats with collagen induced arthritis [24]. In view of the diverse immunomodulatory effects of ginseng polysaccharide, the identification of specific polysaccharides with unique property and biological action will be of great interest.

It appears that AQ and PS ginseng extracts have a paradoxical effect on macrophage function: stimulation under normal condition, but reduction when the biological system is under a pro-inflammatory state. In the context of sepsis, AQ and PS immuno-stimulatory effect will be beneficial as a first line of defense during the initial infection stage of bacterial infection by rendering macrophages to be cytotoxic [25] whereas the immune-suppressive activity may be effective in antagonizing the cytokine storm at the later stages of infection by suppressing LPS activation of macrophages.

Carbohydrates analysis represents a major challenge in analytical chemistry since neutral or acidic saccharides (mono, oligo and poly) have little UV activity. The refractive index (RI) detector, which is commonly used in HPLC analysis, has issues with baseline stability and sensitivity. The ELSD used in the present study has the advantage of its independence of any optical properties in the solutes of interest. Our HPLC-based analysis allowed the measurement of 7 mono-saccharides within a 30 minute total run time. The monosaccharide composition of $P$. quinquefolius reported in this study was similar to that described previously [3] with the addition of galacturonic acid. The monosaccharide composition provides insight into the types of polysaccharides which may be found in P. quinquefolius. Polysaccharides structures in $P$. quinquefolius have not previously been thoroughly studied, though several polysaccharides have been isolated from $P$. ginseng and $P$. notoginseng. Glucose and galacturonic acid were the most prominent monosaccharides detected. Previously, polysaccharide fractions from $P$. ginseng with high levels of glucose have been determined to contain starch-like glucans and arabinogalactans and fractions with high levels of galacturonic acid have been shown to contain pectins with several linked galacturonic acid domains [26]. It is possible that polysaccharides similar to these may be present in Ontario-grown American ginseng though further work will have to be done to characterize their structures. 
Polysaccharides are very complex with a wide range of MW, varying monosaccharide composition and conformation (degree of branching or linearity), which contribute to their diverse structure and biological activities, these can also hamper the study of their structure-function relationships [16]. The multi-detector GPC instrument provided additional information on the polysaccharide structure. This instrument uses Triple detection with a concentration detector (refractive index detector), viscometer and light scattering detector, with each detector providing different although complementary information [20]. Pullulan standards of up to $1,000 \mathrm{kDa}$ showed that the measured ginseng polysaccharide molecular weights for the crude, water soluble and deproteinated extracts are within the range of standards. The light scattering detector, which is based on fluctuations in interference between macromolecules (e.g. polysaccharides) scattering a coherent monochromatic laser beam, is considered an absolute detector for Mw values, confirming the reported values of Table 1 . The viscometer is sensitive to branching effects, although the trace in Figure 9 shows no significant branching effect for the ginseng polysaccharides. The RI detector was rather sensitive to the carbohydrate fractions, showing 3 major polysaccharide components for each of the three measured extracts. The high molecular weight peak has a very low intensity for the PTF sample, indicating that the deproteination step removed the high molecular weight fraction. The neutral extract gave similar molecular weights as the acidic polysaccharide extract, although with a higher sample recovery.

\section{Conclusions and future directions}

Our study has revealed a paradoxical immunomodulatory effect of AQ and PS extracts isolated from Ontario-grown Panax quinquefolius. This activity may be relevant to clinical application involving bacterial infection and toxemia. Challenges in development of botanical polysaccharides for clinical application include lack of methodology for structure identification and characterization, isolation and purification, and product quality control, has been recognized [11]. Our research will focus on the application of new methodologies to characterize the polysaccharide structures from ginseng and to elucidate the structurebiological activity relationship.

\section{Author details}

Edmund M. K. Lui*, Chike G. Azike and Hua Pei

Ontario Ginseng Innovation and Research Consortium

Department of Physiology \& Pharmacology, Schulich School of Medicine and Dentistry, Canada

José A. Guerrero-Analco and John T. Arnason

Ontario Ginseng Innovation and Research Consortium

Department of Biology, University of Ottawa, Ottawa Ontario, Canada

${ }^{*}$ Corresponding Author 
Ahmad A. Romeh

Department of Chemical and Biochemical Engineering, Faculty of Engineering,

University of Western Ontario, London Ontario, Canada

Sherif J. Kaldas

Department of Biology, University of Ottawa, Ottawa Ontario, Canada

Paul A. Charpentier

Ontario Ginseng Innovation and Research Consortium,

Department of Chemical and Biochemical Engineering, Faculty of Engineering,

University of Western Ontario, London Ontario, Canada

\section{Acknowledgement}

This research was supported by Ontario Ginseng Research \& Innovation Consortium (OGRIC) funded by the Ministry of Research \& Innovation, Ontario Research Funded Research Excellence program for the project 'New Technologies for Ginseng Agriculture and Product Development'(RE02-049 awarded to EMK Lui). We acknowledge the contribution of PolyAnalytik London Ontario, Canada in providing instrument for the gel permeation chromatography analysis of ginseng extracts.

\section{References}

[1] Attele AS, Wu JA, Yuan CS (1999) Ginseng Pharmacology; Multiple Constituents and Multiple Actions. Biochem. pharmacol. 58:1685-1693.

[2] Sun Y (2011) Structure and Biological Activities of the Polysaccharides from the Leaves, Roots and Fruits of Panax ginseng C.A. Meyer: An Overview. Carbohyd. polym. 85: 490499.

[3] Assinewe VA, Arnason JT, Aubry A, Mullin J, Lemaire I (2002): Extractable Polysaccharides of Panax quinquefolius L. (North American ginseng) Root Stimulate TNF- $\alpha$ Production by Alveolar Macrophages. Phytomedicine 9:398-404.

[4] Biondo PD, Goruk S, Ruth MR, O'Connell E, Field CJ (2008) Effect of CVT-E002 ${ }^{\mathrm{TM}}$ (COLD-fX®) Versus a Ginsenoside Extract on Systemic and Gut-Associated Immune Function. Int. immunopharmacol. 8:1134-1142.

[5] Predy GN, Goel V, Lovlin RE, Basu TK (2006) Immune Modulating Effects of Daily Supplementation of COLD-FX (a Proprietary Extract of North American Ginseng) in Healthy Adults. J Clin Biochem Nutr 39: 162-167.

[6] Azike CG, Charpentier PA, Hou J, Pei H, Lui EMK. (2011). The Yin and Yang actions of North American ginseng root in modulating the immune function of macrophages Chin Med. 6, 1-12.

[7] Wang M, Guilbert LJ, Li J, Wu Y, Pang P, Basu TK, Shan JJ (2004) A Proprietary Extract from North American Ginseng (Panax quinquefolium) Enhances IL-2 and IFN-gamma Productions in Murine Spleen Cells Induced by Con-A. Int. immunopharmacol. 4:311315. 
[8] Sevag M G, Lackman D B, Smolens J (1938). The isolation of the components of streptococcal nucleoproteins in serologically active form. Journal of Biological Chemistry, 124, 425-436.

[9] Igor A. Schepetkin, Mark T. Quinn (2006). Botanical polysaccharides: Macrophage Immunomodulation \& therapeutic potential. International Immunopharmacology 6: 317-333.

[10] Meiqi Wang, Larry J. Guilbert, Lei Ling, Jie Li, Yingqi Wu, Sharon Xu, Peter Pang and Jacqueline J. Shan (2001). Immunomodulating activity of CVT-E002, a proprietary extract from North American ginseng (Panax quinquefolium). Journal of Pharmacy and Pharmacology 53: 1515-1523.

[11] Jie-Young Song, Seon-Kyu Han, Eun-Hwa Son, Suhk-Neung Pyo, Yeon-Sook Yun, SehYoon, Induction of secretory and tumoricidal activities in peritoneal macrophages by ginsan, Yi International Immunopharmacology 2 (2002) 857-865.

[12] Yoo DG, Kim MC, Park MK, Park KM, Quan FS, Song JM, Wee JJ, Wang BZ, Cho YK, Compans RW, Kang SM (2012). Protective effect of ginseng polysaccharides on influenza viral infection. PLoS One. 2012;7(3):e33678.

[13] Ahn JY, Song JY, Yun YS, Jeong G, Choi IS (2006). Protection of Staphylococcus aureusinfected septic mice by suppression of early acute inflammation and enhanced antimicrobial activity by ginsan. FEMS Immunol Med Microbiol; 46:187-97.

[14] Dianhui Luo, Baishan Fang, Structural identification of ginseng polysaccharides and testing of their antioxidant activities (2008). Carbohydrate Polymers 72 376-381.

[15] Xie JT, Wu JA, Mehendale S, Aung HH, Yuan CS (2004). Anti-hyperglycemic effect of the polysaccharides fraction from American ginseng berry extract in ob/ob mice. Phytomedicine. 11:182-7.

[16] He X, Niu X, Li J, Xu S , Lu A (2012). Immunomodulatory Activities of Five Clinically Used Chinese Herbal Polysaccharides. J. exp. integr. med. 2:15-27.

[17] Wu Y, Lu X, Xiang FL, Lui EMK, Feng Q (2011) Ginseng protects the heart from ischemia and reperfusion injury via up regulation of endothelial nitric oxide synthase. Pharmacological Research. 64:195-202.

[18] Subhrojit S, Chen S, Feng B, Wu Y, Lui E, Chakrabarti S (2012) Preventive effects of North American ginseng (Panax quinquefolius) on diabetic retinopathy and cardiomyopathy. Phytotheraphy Res. (In Press)

[19] Subhrojit S, Chen S, Feng B, Wu Y, Lui E, Chakrabarti S (2012) Preventive effects of North American ginseng (Panax quinquefolius) on diabetic neuropathy. Phytomedicine. 19: 494- 505.

[20] Zhou D, Tong L, Wan M, Wang G, Ye Z, Wang Z, Lin R (2011). An LC-MS method for simultaneous determination of nine ginsenosides in rat plasma and its application in pharmacokinetic study. Biomed Chromatogr, 25::720-6.

[21] Lee DC, Yang CL, Chik SC, Li JC, Rong JH, Chan GC, Lau AS (2009) Bioactivity-Guided Identification and Cell Signalling Technology to Delineate the Immunomodulatory Effects of Panax ginseng on Human Promonocytic U937 cells. J. transl. med. 34:1-10.

[22] Oh GS, Pae HO, Choi BM, Seo EA, Kim DH, Shin MK, Kim JD, Kim JB, Chung HT (2004). 20(S)-Protopanaxatriol, one of ginsenoside metabolites, inhibits inducible nitric 
oxide synthase and cyclooxygenase-2 expressions through inactivation of nuclear factor-kappaB in RAW 264.7 macrophages stimulated with lipopolysaccharide. Cancer Lett, 205:23-29.

[23] Leon-Ponte M, Kirchhof MG, Sun T, Stephens T, Singh B, Sandhu S, Madrenas J. (2005). Polycationic lipids inhibit the pro-inflammatory response to LPS. Immunol Lett. 96:7383.

[24] Zhao H, Zhang W, Xiao C, Lu C, Xu S, He X, Li X, Chen S, Yang D, Chan ASC, Lu A (2011) Effect of Ginseng Polysaccharide on TNF- $\alpha$ and INF- $\gamma$ Produced by Enteric Mucosal Lymphocytes in Collagen Induced Arthritic Rats. J. med. plants res. 5:15361542.

[25] Lim DS, Bae KG, Jung IS, Kim CH, Yun YS, Song JY (2002) Anti-Septicaemic Effect of Polysaccharide from Panax ginseng by Macrophage Activation. J. infection. 45: 32-38.

[26] Zhang X, Yu L, Bi H, Li X, Ni W, Han H, Li N, Wang B, Zhou Y, Tai G (2009) Total Fractionation and characterization of the Water-Soluble Polysaccharides Isolated from Panax ginseng C.A. Meyer. Carbohyd. polym. 77: 544-552. 